\title{
The gamma-ray emission from 3HWC J1928+178
}

\section{Armelle Jardin-Blicq* on behalf of the HAWC Collaboration}

(a complete list of authors can be found at the end of the proceedings)

National Astronomical Research Institute of Thailand (Public Organization), Don Kaeo, MaeRim, Chiang Mai 50180, Thailand

Department of Physics, Faculty of Science, Chulalongkorn University, 254 Phayathai Road, Pathumwan, Bangkok 10330, Thailand

Max-Planck Institute for Nuclear Physics, D-69117 Heidelberg, Germany

E-mail: armelle@narit.or.th

The gamma-ray source 3HWC J1928+178, discovered by HAWC, is coincident with the $82 \mathrm{kyr}$ pulsar PSR J1928+1746, located $4 \mathrm{kpc}$ away. It has not been reported by any Imaging atmospheric Cherenkov Telescope (IACT), until the recent detection of emission from this region by HESS, using an analysis adapted to extended sources. No counterpart in GeV gamma-rays from FermiLAT data or in X-ray has been reported so far. In this contribution, I give the multiwavelength context of the region surrounding 3HWC J1928+178 and present a multi-component model derived using the Multi-Mission Maximum Likelihood framework (3ML). I explore the possibility to model the gamma-ray emission of 3HWC J1928+178 by an extended source with continuous diffuse emission. Together with the age of the pulsar and its extended nature, it may indicate a transition from a pulsar wind nebulae to a halo, where the electrons have started to cool and diffuse away from the source.

$37^{\text {th }}$ International Cosmic Ray Conference (ICRC 2021)

July 12th - 23rd, 2021

Online - Berlin, Germany

\footnotetext{
*Presenter
} 


\section{Introduction}

\subsection{Astrophysical context}

3HWC $\mathrm{J} 1928+178\left(\ell=52.93^{\circ}, b=0.2^{\circ}\right)$ is a gamma-ray source discovered by HAWC and reported in the $3 \mathrm{HWC}$ catalogue [1] at the significance level of $\sim 15 \sigma$. No Imaging Atmospheric Cherenkov Telescope (IACT) has detected it before the confirmation by the H.E.S.S. collaboration[2], using an analysis method adapted to extended sources. The origin of the observed very high energy gamma-ray emission is still unclear. It may be associated with the pulsar PSR J1928+1746, discovered at radio wavelength [3], although neither the pulsar nor the pulsar wind nebulae (PWN) has been detected in the X-ray energy range. In the vicinity of 3HWC J1928+178 is another HAWC source, 3HWC J1930+188 $\left(\ell=54.03^{\circ}, b=0.32^{\circ}\right)$, also detected by H.E.S.S. [4] and VERITAS [5], associated with the PWN in the supernova remnant SNR G54.1+0.3. It hosts the pulsar PSR 1930+1852. Finally, another energetic pulsar is located nearby, PSR J1932+1916, associated with the Fermi source 3FGL J1932.2+1916. The characteristics of the three pulsars are gathered in Table 1.

Table 1: Characteristics of the pulsars located in the vicinity of 3HWC J1928+178 taken from the ATNF catalogue [6].

\begin{tabular}{|c|c|c|c|}
\hline & PSR J1928+1746 & PSR J1930+1852 & PSR J1932+1916 \\
\hline${\text { celestial coordinates }\left(\mathrm{ra}^{\circ}, \mathrm{dec}^{\circ}\right)}^{\circ}(292.18,17.77)$ & $(292.62,18.87)$ & $(293.08,19.28)$ \\
\hline${\text { galactic coordinates }\left(\mathrm{l}^{\circ}, \mathrm{b}^{\circ}\right)}^{\circ}$ & $(52.93,0.11)$ & $(54.1,0.26)$ & $(54.67,0.09)$ \\
\hline distance $(\mathrm{kpc})$ & 4.3 & 6.2 & - \\
\hline age $(\mathrm{kyr})$ & 82.6 & 2.9 & 35.4 \\
\hline period $(\mathrm{s})$ & 0.069 & 0.14 & 0.21 \\
\hline spin down power $\left(\mathrm{erg} \mathrm{s}^{-1}\right)$ & $1.6 \times 10^{36}$ & $1.2 \times 10^{37}$ & $4.1 \times 10^{35}$ \\
\hline
\end{tabular}

\subsection{The HAWC observatory and HAWC Data}

The High Altitude Water Cherenkov (HAWC) gamma-ray observatory is located at a latitude of $19^{\circ} \mathrm{N}$ in Mexico, and at $4100 \mathrm{~m}$ in altitude. HAWC is composed of 300 water tanks instrumented with four photomultiplier tubes (PMT). When the secondary particles of an atmospheric air shower passes through HAWC, they produce Cherenkov light in the water tanks and each PMT records the time and amplitude of the signal. Combining the information of all the PMTs, we can build the footprint of the shower on the detector and reconstruct the parameters of the air shower. Each event is assigned to one of the 9 analysis bin depending on the fraction of the water tanks that has been hit. Bin definitions are given in [7]. For this analysis, 1523 days of HAWC data are used and events falling in the analysis bins 4 to 9 are selected. It corresponds to events triggering more than $25 \%$ of the array, which gives an energy threshold of approximately $1 \mathrm{TeV}$. For this specific bin selection, the sources 3HWC J1928+178 is refered to as HAWC J1928+178 in [2]. The corresponding PSF of the instrument is $\sim 0.4^{\circ}$ for bin 4 and decreases to less than $0.2^{\circ}$ for bin 9 . More information about the detector, the event reconstruction and the data analysis can be found in [8] and [7]. 


\section{Modeling the region around $3 H W C$ J1928+178}

A multi-component fit based on a maximum likelihood approach is performed using the MultiMission Maximum Likelihood framework (3ML) [9] and the HAWC HAL ${ }^{1}$ plugin. A model is defined for a $3.5^{\circ}$ radius region around 3 HWC J1928+178, convolved with the instrument response and compared to the corresponding experimental data. Two models are considered, described in the following paragraphs.

\subsection{The four components model}

Following an iterative procedure, a first model is defined composed of 2 components: a point-like component and an extended symmetric Gaussian component at the location of the two HAWC sources 3HWC J1930+188 and 3HWC 1928+178 respectively. This choice is motivated by the fact that 3 HWC J1930+188 was detected as a point-like source by HAWC, H.E.S.S. [4] and VERITAS [5], and by previous studies of 3HWC J1928+178 that showed that it is likely extended [2] [10]. A simple power law $\mathrm{dN} / \mathrm{dE}=\mathrm{F}_{0}\left(\mathrm{E} / \mathrm{E}_{0}\right)^{\Gamma}$ is assumed as energy spectrum for all components. The fit is performed with the position, size of the extended source, flux normalisation $\mathrm{F}_{0}$ at $\mathrm{E}_{0}=10 \mathrm{TeV}$ and spectral index $\Gamma$ as free parameters. For each model, a test statistic (TS) is computed that compares the likelihood that the region is represented by the model against the hypothesis that there is background fluctuations only: $\mathrm{TS}=2 \ln (\mathcal{L}$ (model) $/ \mathcal{L}$ (background)). According to Wilks' theorem, the quantity TS follows a $\chi^{2}$ distribution of $\mathrm{N}$ degrees of freedom, with $\mathrm{N}$ the difference in number of free parameters between the model and the background [11]. If an excess is found in the residual maps, a new component is added at the location of the excess and the fit is performed again with the additional component. The procedure stops when the addition of a new component does not improve the fit by more than $\triangle \mathrm{TS}=25$. The best model is found to be made of two point-like sources close to the location of the pulsars PSR J1930+1852 and PSR J1932+1916, and two extended sources represented by symmetric Gaussians. The first one is found to have a size $\sigma=0.18^{\circ}$ (39\% containment) and is located at the position of 3HWC J1928+178. The second one is very extended with a size $\sigma=1.43^{\circ}$ and seems to cover the whole region, likely trying to account for some large scale gamma-ray emission, maybe diffuse emission from the galactic plane. The output parameters can be found in Table 2 and the model can be visualised on the top right panel of Figure 1.

\subsection{The diffusion emission model}

Alternatively, a diffusion model is considered for 3HWC J1928+178, assuming a continuous injection of electrons and positrons at the location of the remaining excess for 3HWC J1928+178 after the first fit. Similar to the Geminga analysis [12], it is motivated by the fact that PSR J1928+1746 is a rather old pulsar, that we don't see any X-ray counterpart and that the $\gamma$-ray emission seems extended. Indeed, the four components model shows that 3HWC J1928+178 seems to be described by a superposition of a small Gaussian on top of a very wide one, which are both incorporated in this diffuse component for 3HWC J1928+178. This model is thus composed of three components only: two point-like and one extended component with diffuse emission. As described in [12], the $\gamma$-ray

\footnotetext{
${ }^{1}$ https://github.com/threeML/hawc_hal
} 
flux $f_{d}$ for $3 \mathrm{HWC} \mathrm{J} 1928+178$, as a function of the distance from the source $d$, is approximately equal to:

$$
f_{d}=\frac{1.22}{\pi^{3 / 2} r_{d}\left(d+0.06 r_{d}\right)} \exp \frac{-d^{2}}{r_{d}^{2}}
$$

where $r_{d}$ is the diffusion radius that is a free parameter fitted together with the position of the three components and their spectral parameter. The diffusion radius is found to be $2.68^{\circ}$. All the output parameters are gathered in Table 2 and the model can be visualised on the bottom right panel of Figure 1.
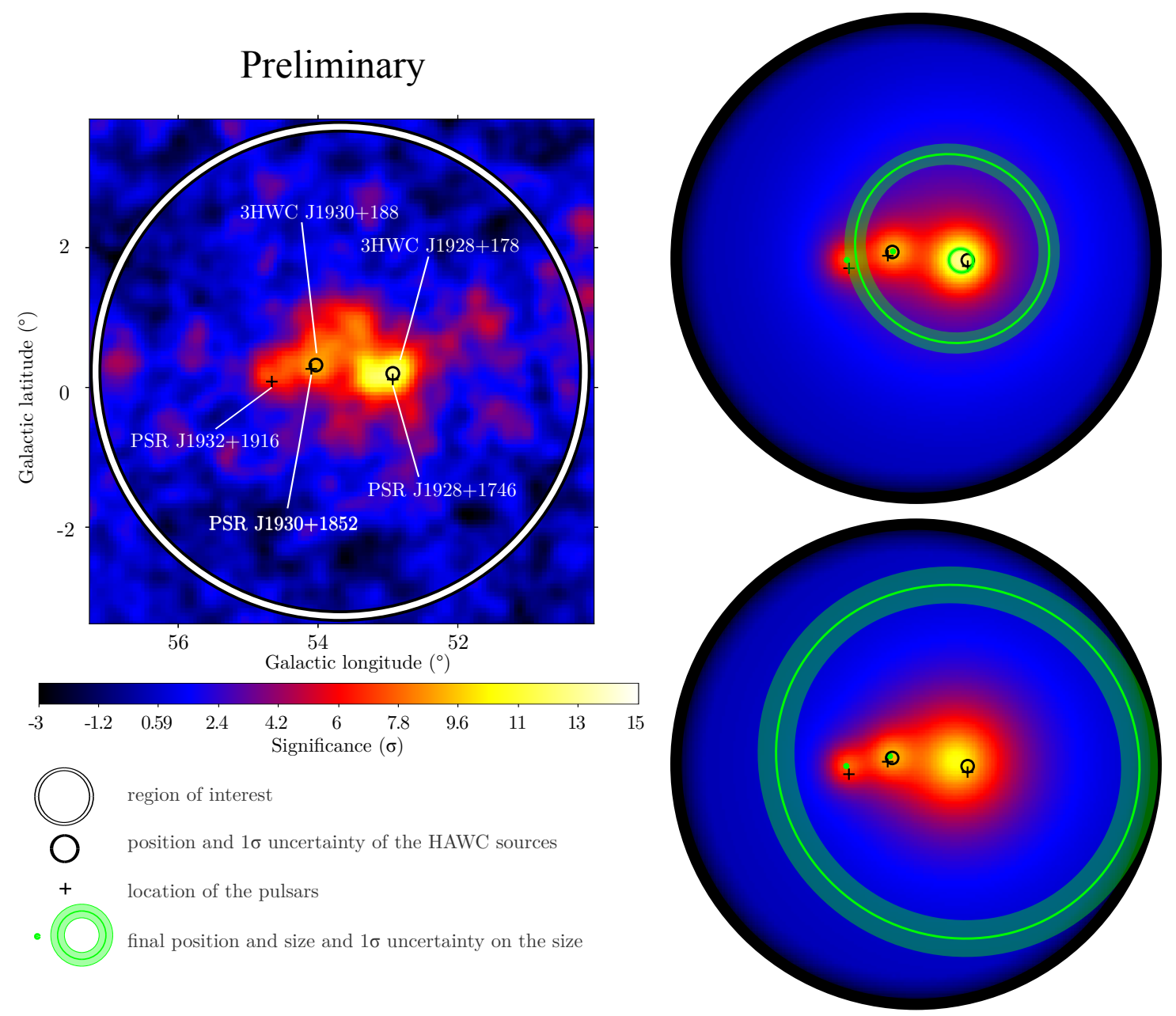

Figure 1: Left: HAWC significance map with the location of the HAWC sources and the location of the pulsars. The white circle is the region of interest of $3.5^{\circ}$ radius. Right: Significance map of the 4 components model at the top and the diffusion model at the bottom, with the position and size of each fitted components. Each map is $3.5^{\circ}$ in radius

\section{Comparison between the 2 models}

The diffusion model is slightly worse by $\Delta \mathrm{TS}=8$ than the model with 4 components ( 2 pointlike and 2 extended sources) but it has less degrees of freedom since it has only 3 components. To 
Table 2: Output values from the fit for the components of the two different models representing 3HWC J1928+178.

\begin{tabular}{|c|c|c|c|}
\hline & \multicolumn{2}{|c|}{ 4 components model } & diffusion model \\
\hline hypothesis & Gaussian 1 & Gaussian 2 & continuous injection \\
\hline$\left(\mathrm{ra}^{\circ}, \mathrm{dec}^{\circ}\right)$ & $\left(292.15_{ \pm 0.03}, 17.90_{ \pm 0.04}\right)$ & $\left(292.05_{ \pm 0.15}, 18.10_{ \pm 0.17}\right)$ & $\left(292.10_{ \pm 0.06}, 17.98_{ \pm 0.02}\right)$ \\
\hline size $\left(\sigma\right.$ or $\left.r_{d}\right)\left(^{\circ}\right)$ & $0.18_{ \pm 0.03}$ & $1.43_{ \pm 0.16}$ & $2.68_{ \pm 0.27}$ \\
\hline index & $-2.09_{ \pm 0.15}$ & $-2.60_{ \pm 0.08}$ & $-2.58_{ \pm 0.05}$ \\
\hline flux $_{10 \mathrm{TeV}}\left(\times 10^{-15}\right.$ & $4.2_{-1.1}^{+1.5}$ & $40_{-4}^{+5}$ & $47_{-5}^{+5}$ \\
$\left.\mathrm{TeV}^{-1} \mathrm{~cm}^{-2} \mathrm{~s}^{-1}\right)$ & & & \\
\hline
\end{tabular}

account for the difference in number of degrees of freedom we can look at the Bayesian information criterion number (BIC) for the two non-nested models used here [13], given by this formula : $\mathrm{BIC}=-2 \ln (\mathcal{L})+k \ln (n)$ where $k$ is the number of free parameters and $n$ is the number of healpix pixels in the ROI. It seems that the diffusion model is largely preferred with a difference of $\Delta \mathrm{BIC}=45$. Figure 2 shows the radial profiles centered on $3 \mathrm{HWC}$ J1928+178 of the HAWC data, and for the two models. The profile of the Crab nebula is plotted as a reference for the HAWC PSF, showing that the emission is indeed extended. The profiles of both models follow closely the data.

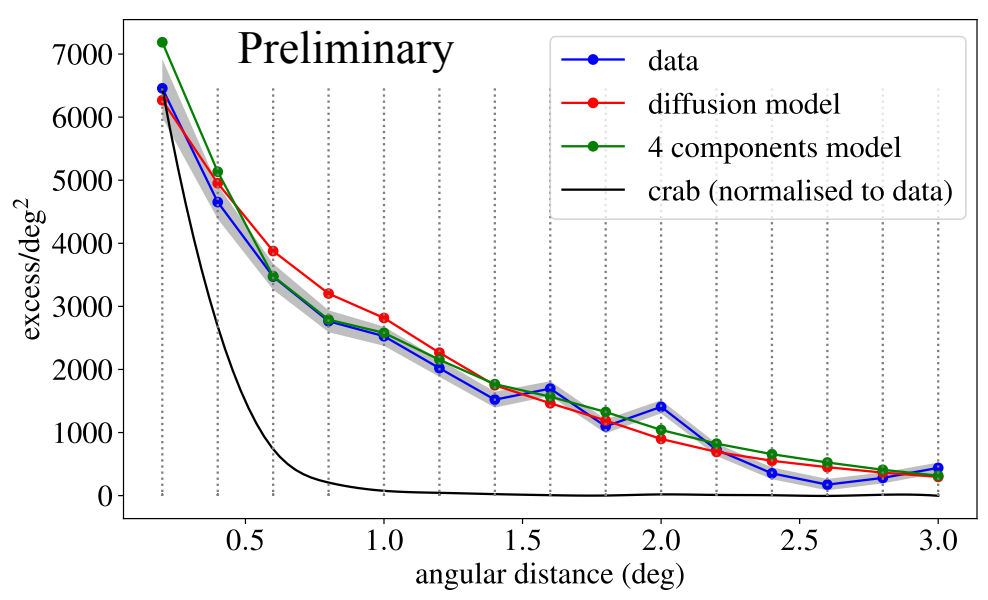

Figure 2: Radial profile centered on $\mathrm{J} 1928$ in step of $0.2^{\circ}$, for the HAWC data (blue) with its standard deviation (grey band), the diffuse model (red) and the 4 components model (green). The profile of the models is plotted for the region of interest of $2^{\circ}$ only. The profile of the crab nebula is also plotted (black) as a reference for the HAWC PSF.

The spectral energy distribution is plotted in Figure 3 for the component representing the source 3 HWC J1928+178 for the two models. It is compared with the spectrum from the HAWC automatic source search for the same set of data [2]. The flux point from LHAASO [14] seems to be more compatible with the 4 components model, or imply a cut-off in the power low spectrum from the diffusion model. 


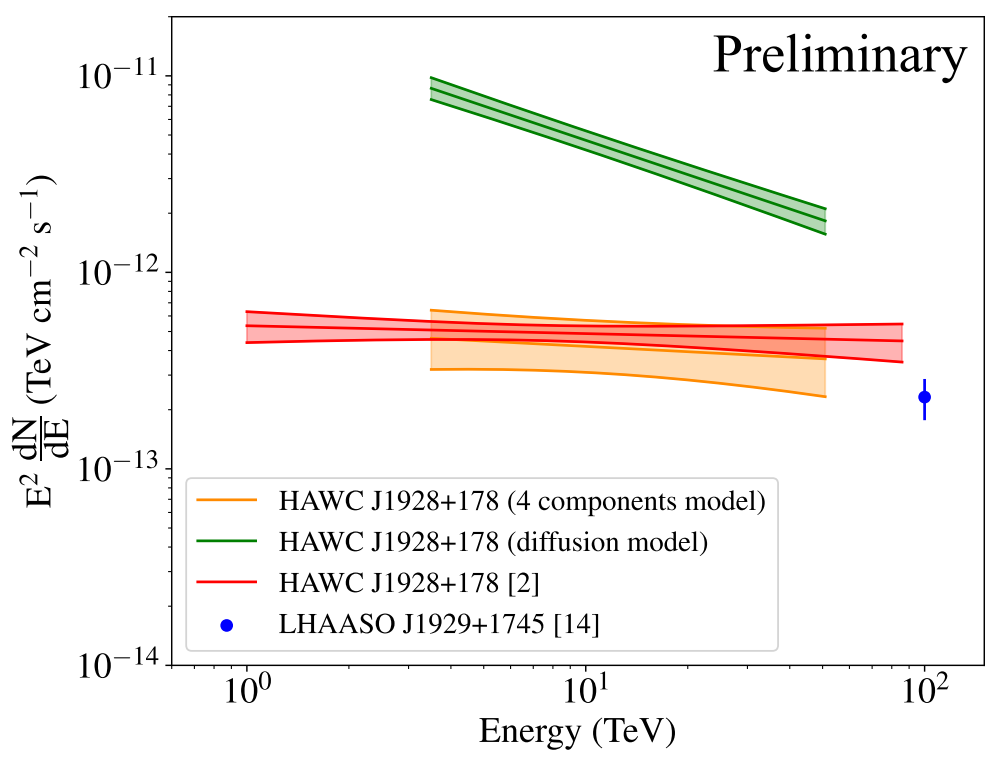

Figure 3: Spectral energy distribution of 3HWC J1928+178 for the two models presented in this contribution, for the energy range $3.5-51 \mathrm{TeV}$, corresponding to the median energies of bin 4 and 9 . The spectrum from the HAWC catalogue search for the same analysis bins [2] and the flux point from LHAASO are also plotted for comparison [14]. The shaded area correspond to the $1 \sigma$ statistical uncertainties.

\section{Discussion and conclusion}

The 4 components model and the diffusion model are very similar in the sense that they are in

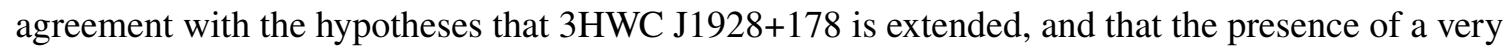
large scale component is needed, either maybe due the galactic diffuse emission in one case, or to the diffusion of $\mathrm{e}^{ \pm}$in the second case. The diffusion model, with 1 component less and a lower BIC, seemed favoured. It may indicate that $3 \mathrm{HWC} \mathrm{J} 1928+178$ is in a transition phase to a $\mathrm{TeV}$ gamma-ray halo: the pulsar being rather old, the $\mathrm{TeV}$ gamma-ray emission being extended, and the fact that no PWN has been detected in X-ray are favouring this hypothesis. However, the fitted diffusion radius $r_{d}$ is found to be $2.68^{\circ}$, i.e $\sim 400 \mathrm{pc}$ in diameter, since PSR J1928+1746 is located $4.3 \mathrm{kpc}$ away. It is huge compared to Geminga which has a diffusion radius of $5.5^{\circ}$ but is only 250 pc away from us, giving a size of $23 \mathrm{pc}$ in diameter. This may disfavour this model. Moreover, the spectrum for 3 HWC J1928+178 from the 4 components model is in better agreement with the measurement from LHAASO. A deeper study at the highest energy, using the energy estimators developed by the HAWC collaboration [15] instead of the analysis bins would help to clarify the presence of a cut-off.

\section{Acknowledgments}

We acknowledge the support from: the US National Science Foundation (NSF); the US Department of Energy Office of High-Energy Physics; the Laboratory Directed Research and Development (LDRD) program of Los Alamos National Laboratory; Consejo Nacional de Ciencia y Tecnología (CONACyT), México, grants 271051, 232656, 260378, 179588, 254964, 258865, 243290, 132197 , 
A1-S-46288, A1-S-22784, cátedras 873, 1563, 341, 323, Red HAWC, México; DGAPA-UNAM grants IG101320, IN111716-3, IN111419, IA102019, IN110621, IN110521; VIEP-BUAP; PIFI 2012, 2013, PROFOCIE 2014, 2015; the University of Wisconsin Alumni Research Foundation; the Institute of Geophysics, Planetary Physics, and Signatures at Los Alamos National Laboratory; Polish Science Centre grant, DEC-2017/27/B/ST9/02272; Coordinación de la Investigación Científica de la Universidad Michoacana; Royal Society - Newton Advanced Fellowship 180385; Generalitat Valenciana, grant CIDEGENT/2018/034; Chulalongkorn University's CUniverse (CUAASC) grant; Coordinación General Académica e Innovación (CGAI-UdeG), PRODEP-SEP UDG-CA499; Institute of Cosmic Ray Research (ICRR), University of Tokyo, H.F. acknowledges support by NASA under award number 80GSFC21M0002. We also acknowledge the significant contributions over many years of Stefan Westerhoff, Gaurang Yodh and Arnulfo Zepeda Dominguez, all deceased members of the HAWC collaboration. Thanks to Scott Delay, Luciano Díaz and Eduardo Murrieta for technical support.

\section{References}

[1] A. Albert, R. Alfaro, C. Alvarez, J.R.A. Camacho, J.C. Arteaga-Velázquez, K.P. Arunbabu et al., 3HWC: The Third HAWC Catalog of Very-high-energy Gamma-Ray Sources, Astrophysical Journal 905 (2020) 76 [2007.08582].

[2] H. Abdalla, F. Aharonian, F.A. Benkhali, E.O. Angüner, C. Arcaro, C. Armand et al., Tev emission of galactic plane sources with HAWC and H.E.S.S., 2021.

[3] J.M. Cordes, P.C.C. Freire, D.R. Lorimer, F. Camilo, D.J. Champion, D.J. Nice et al., Arecibo Pulsar Survey Using ALFA. I. Survey Strategy and First Discoveries, The Astrophysical Journal 637 (2006) 446 [astro-ph/0509732].

[4] H. E. S. S. Collaboration, H. Abdalla, A. Abramowski, F. Aharonian, F.A. Benkhali, E.O. Angüner et al., The H.E.S.S. Galactic plane survey, A\&A 612 (2018) A1 [1804 . 02432].

[5] V.A. Acciari, E. Aliu, T. Arlen, T. Aune, M. Bautista, M. Beilicke et al., Discovery of Very High Energy $\gamma$-ray Emission from the SNR G54.1+0.3, ApJL 719 (2010) L69 [1005 . 0032].

[6] R.N. Manchester, G.B. Hobbs, A. Teoh and M. Hobbs, The australia telescope national facility pulsar catalogue, The Astrophysical Journal 129 (2005) 1993.

[7] A.U. Abeysekara, A. Albert, R. Alfaro, C. Alvarez, J.D. Álvarez, R. Arceo et al., Observation of the Crab Nebula with the HAWC Gamma-Ray Observatory, ApJ 843 (2017) 39 [1701.01778].

[8] A.J. Smith and HAWC Collaboration, HAWC: Design, Operation, Reconstruction and Analysis, in 34th International Cosmic Ray Conference (ICRC2015), vol. 34 of International Cosmic Ray Conference, p. 966, July, 2015 [1508.05826].

[9] G. Vianello, R.J. Lauer, P. Younk, L. Tibaldo, J.M. Burgess, H. Ayala et al., The Multi-Mission Maximum Likelihood framework (3ML), Proceedings of the 34th ICRC, The Hague, Netherlands (2015) [1507.08343]. 
[10] R. López-Coto, V. Marandon, F. Brun, for the HAWC collaboration and the HESS collaboration, Morphological and spectral measurements of 2 HWC J1928+177 with HAWC and H.E.S.S., Proceedings of the 35th ICRC, Busan, Korea (2017) .

[11] S.S. Wilks, The Large-Sample Distribution of the Likelihood Ratio for Testing Composite Hypotheses, The Annals of Mathematical Statistics 9 (1938) 60 .

[12] A.U. Abeysekara, A. Albert, R. Alfaro, C. Alvarez, J.D. Álvarez, R. Arceo et al., Extended gamma-ray sources around pulsars constrain the origin of the positron flux at Earth, Science 358 (2017) 911 [1711.06223].

[13] G. Schwarz, Estimating the Dimension of a Model, The Annals of Statistics 6 (1978) 461.

[14] Z. Cao, F. Aharonian, Q. An, Axikegu, L.X. Bai, Y.X. Bai et al., Ultrahigh-energy photons up to 1.4 petaelectronvolts from 12 gamma-ray Galactic sources, Nature 594 (2021) 33-36.

[15] A.U. Abeysekara, A. Albert, R. Alfaro, C. Alvarez, J.D. Álvarez, J.R.A. Camacho et al., Measurement of the Crab Nebula Spectrum Past $100 \mathrm{TeV}$ with HAWC, The astrophysical Journal 881 (2019) 134 [1905. 12518]. 


\section{Full Authors List: HAWC Collaboration}

A.U. Abeysekara ${ }^{48}$, A. Albert ${ }^{21}$, R. Alfaro ${ }^{14}$, C. Alvarez $^{41}$, J.D. Álvarez ${ }^{40}$, J.R. Angeles Camacho ${ }^{14}$, J.C. Arteaga-Velázquez ${ }^{40}$, K. P. Arunbabu $^{17}$, D. Avila Rojas ${ }^{14}$, H.A. Ayala Solares ${ }^{28}$, R. Babu ${ }^{25}$, V. Baghmanyan ${ }^{15}$, A.S. Barber ${ }^{48}$, J. Becerra Gonzalez ${ }^{11}$, E. BelmontMoreno $^{14}$, S.Y. BenZvi ${ }^{29}$, D. Berley ${ }^{39}$, C. Brisbois ${ }^{39}$, K.S. Caballero-Mora ${ }^{41}$, T. Capistrán ${ }^{12}$, A. Carramiñana ${ }^{18}$, S. Casanova ${ }^{15}$, O. Chaparro-Amaro $^{3}$, U. Cotti ${ }^{40}$, J. Cotzomi ${ }^{8}$, S. Coutiño de León ${ }^{18}$, E. De la Fuente ${ }^{46}$, C. de León ${ }^{40}$, L. Diaz-Cruz ${ }^{8}$, R. Diaz Hernandez ${ }^{18}$, J.C. Díaz-Vélez ${ }^{46}$, B.L. Dingus ${ }^{21}$, M. Durocher ${ }^{21}$, M.A. DuVernois ${ }^{45}$, R.W. Ellsworth ${ }^{39}$, K. Engel ${ }^{39}$, C. Espinoza ${ }^{14}$, K.L. Fan ${ }^{39}$, K. Fang $^{45}$, M. Fernández Alonso ${ }^{28}$, B. Fick ${ }^{25}$, H. Fleischhack ${ }^{51,11,52}$, J.L. Flores ${ }^{46}$, N.I. Fraija ${ }^{12}$, D. Garcia ${ }^{4}$, J.A. García-González ${ }^{20}$, J. L. García-Luna ${ }^{46}$, G. García-Torales ${ }^{46}$, F. Garfias ${ }^{12}$, G. Giacinti ${ }^{22}$, H. Goksu ${ }^{22}$, M.M. González ${ }^{12}$, J.A. Goodman ${ }^{39}$, J.P. Harding ${ }^{21}$, S. Hernandez ${ }^{14}$, I. Herzog ${ }^{25}$, J. Hinton ${ }^{22}$, B. Hona ${ }^{48}$, D. Huang ${ }^{25}$, F. Hueyotl-Zahuantitla ${ }^{41}$, C.M. Hui ${ }^{23}$, B. Humensky ${ }^{39}$, P. Hüntemeyer ${ }^{25}$, A. Iriarte ${ }^{12}$, A. Jardin-Blicq ${ }^{22,49,50}$, H. Jhee ${ }^{43}$, V. Joshi ${ }^{7}$, D. Kieda ${ }^{48}$, G J. Kunde ${ }^{21}$, S. Kunwar ${ }^{22}$, A. Lara ${ }^{17}$, J. Lee ${ }^{43}$, W.H. Lee ${ }^{12}$, D. Lennarz ${ }^{9}$, H. León Vargas ${ }^{14}$, J. Linnemann ${ }^{24}$, A.L. Longinotti ${ }^{12}$, R. López-Coto ${ }^{19}$, G. Luis-Raya ${ }^{44}$, J. Lundeen ${ }^{24}$, K. Malone ${ }^{21}$, V. Marandon $^{22}$, O. Martinez ${ }^{8}$, I. Martinez-Castellanos ${ }^{39}$, H. Martínez-Huerta ${ }^{38}$, J. Martínez-Castro ${ }^{3}$, J.A.J. Matthews ${ }^{42}$, J. McEnery ${ }^{11}$, P. Miranda-Romagnoli $^{34}$, J.A. Morales-Soto ${ }^{40}$, E. Moreno ${ }^{8}$, M. Mostafá ${ }^{28}$, A. Nayerhoda ${ }^{15}$, L. Nellen ${ }^{13}$, M. Newbold ${ }^{48}$, M.U. Nisa ${ }^{24}$, R. Noriega-Papaqui ${ }^{34}$, L. Olivera-Nieto ${ }^{22}$, N. Omodei ${ }^{32}$, A. Peisker ${ }^{24}$, Y. Pérez Araujo ${ }^{12}$, E.G. Pérez-Pérez ${ }^{44}$, C.D. Rho ${ }^{43}$, C. Rivière ${ }^{39}$, D. Rosa-Gonzalez ${ }^{18}$, E. Ruiz-Velasco ${ }^{22}$, J. Ryan ${ }^{26}$, H. Salazar ${ }^{8}$, F. Salesa Greus ${ }^{15,53}$, A. Sandoval ${ }^{14}$, M. Schneider ${ }^{39}$, H. Schoorlemmer ${ }^{22}$, J. Serna-Franco ${ }^{14}$, G. Sinnis ${ }^{21}$, A.J. Smith ${ }^{39}$, R.W. Springer ${ }^{48}$, P. Surajbali ${ }^{22}$, I. Taboada ${ }^{9}$, M. Tanner ${ }^{28}$, K. Tollefson ${ }^{24}$, I. Torres ${ }^{18}$, R. Torres-Escobedo $^{30}$, R. Turner ${ }^{25}$, F. Ureña-Mena ${ }^{18}$, L. Villaseñor ${ }^{8}$, X. Wang ${ }^{25}$, I.J. Watson ${ }^{43}$, T. Weisgarber ${ }^{45}$, F. Werner ${ }^{22}$, E. Willox ${ }^{39}$, J. Wood ${ }^{23}$, G.B. Yodh ${ }^{35}$, A. Zepeda ${ }^{4}$, H. Zhou ${ }^{30}$

${ }^{1}$ Barnard College, New York, NY, USA, ${ }^{2}$ Department of Chemistry and Physics, California University of Pennsylvania, California, PA, USA, ${ }^{3}$ Centro de Investigación en Computación, Instituto Politécnico Nacional, Ciudad de México, México, ${ }^{4}$ Physics Department, Centro de Investigación y de Estudios Avanzados del IPN, Ciudad de México, México, ${ }^{5}$ Colorado State University, Physics Dept., Fort Collins, CO, USA, ${ }^{6}$ DCI-UDG, Leon, Gto, México, ${ }^{7}$ Erlangen Centre for Astroparticle Physics, Friedrich Alexander Universität, Erlangen, BY, Germany, ${ }^{8}$ Facultad de Ciencias Físico Matemáticas, Benemérita Universidad Autónoma de Puebla, Puebla, México, ${ }^{9}$ School of Physics and Center for Relativistic Astrophysics, Georgia Institute of Technology, Atlanta, GA, USA, ${ }^{10}$ School of Physics Astronomy and Computational Sciences, George Mason University, Fairfax, VA, USA, ${ }^{11}$ NASA Goddard Space Flight Center, Greenbelt, MD, USA, ${ }^{12}$ Instituto de Astronomía, Universidad Nacional Autónoma de México, Ciudad de México, México, ${ }^{13}$ Instituto de Ciencias Nucleares, Universidad Nacional Autónoma de México, Ciudad de México, México, ${ }^{14}$ Instituto de Física, Universidad Nacional Autónoma de México, Ciudad de México, México, ${ }^{15}$ Institute of Nuclear Physics, Polish Academy of Sciences, Krakow, Poland, ${ }^{16}$ Instituto de Física de São Carlos, Universidade de São Paulo, São Carlos, SP, Brasil, ${ }^{17}$ Instituto de Geofísica, Universidad Nacional Autónoma de México, Ciudad de México, México, ${ }^{18}$ Instituto Nacional de Astrofísica, Óptica y Electrónica, Tonantzintla, Puebla, México, ${ }^{19}$ INFN Padova, Padova, Italy, ${ }^{20}$ Tecnologico de Monterrey, Escuela de Ingeniería y Ciencias, Ave. Eugenio Garza Sada 2501, Monterrey, N.L., 64849, México, ${ }^{21}$ Physics Division, Los Alamos National Laboratory, Los Alamos, NM, USA, ${ }^{22}$ Max-Planck Institute for Nuclear Physics, Heidelberg, Germany, ${ }^{23}$ NASA Marshall Space Flight Center, Astrophysics Office, Huntsville, AL, USA, ${ }^{24}$ Department of Physics and Astronomy, Michigan State University, East Lansing, MI, USA, ${ }^{25}$ Department of Physics, Michigan Technological University, Houghton, MI, USA, ${ }^{26}$ Space Science Center, University of New Hampshire, Durham, NH, USA, ${ }^{27}$ The Ohio State University at Lima, Lima, OH, USA, ${ }^{28}$ Department of Physics, Pennsylvania State University, University Park, PA, USA, ${ }^{29}$ Department of Physics and Astronomy, University of Rochester, Rochester, NY, USA, ${ }^{30}$ Tsung-Dao Lee Institute and School of Physics and Astronomy, Shanghai Jiao Tong University, Shanghai, China, ${ }^{31}$ Sungkyunkwan University, Gyeonggi, Rep. of Korea, ${ }^{32}$ Stanford University, Stanford, CA, USA, ${ }^{33}$ Department of Physics and Astronomy, University of Alabama, Tuscaloosa, AL, USA, ${ }^{34}$ Universidad Autónoma del Estado de Hidalgo, Pachuca, Hgo., México, ${ }^{35}$ Department of Physics and Astronomy, University of California, Irvine, Irvine, CA, USA, ${ }^{36}$ Santa Cruz Institute for Particle Physics, University of California, Santa Cruz, Santa Cruz, CA, USA, ${ }^{37}$ Universidad de Costa Rica, San José, Costa Rica, ${ }^{38}$ Department of Physics and Mathematics, Universidad de Monterrey, San Pedro Garza García, N.L., México, ${ }^{39}$ Department of Physics, University of Maryland, College Park, MD, USA, ${ }^{40}$ Instituto de Física y Matemáticas, Universidad Michoacana de San Nicolás de Hidalgo, Morelia, Michoacán, México, ${ }^{41}$ FCFM-MCTP, Universidad Autónoma de Chiapas, Tuxtla Gutiérrez, Chiapas, México, ${ }^{42}$ Department of Physics and Astronomy, University of New Mexico, Albuquerque, NM, USA, ${ }^{43}$ University of Seoul, Seoul, Rep. of Korea, ${ }^{44}$ Universidad Politécnica de Pachuca, Pachuca, Hgo, México, ${ }^{45}$ Department of Physics, University of Wisconsin-Madison, Madison, WI, USA, ${ }^{46}$ CUCEI, CUCEA, Universidad de Guadalajara, Guadalajara, Jalisco, México, ${ }^{47}$ Universität Würzburg, Institute for Theoretical Physics and Astrophysics, Würzburg, Germany, ${ }^{48}$ Department of Physics and Astronomy, University of Utah, Salt Lake City, UT, USA, ${ }^{49}$ Department of Physics, Faculty of Science, Chulalongkorn University, Pathumwan, Bangkok 10330, Thailand, ${ }^{50}$ National Astronomical Research Institute of Thailand (Public Organization), Don Kaeo, MaeRim, Chiang Mai 50180, Thailand, ${ }^{51}$ Department of Physics, Catholic University of America, Washington, DC, USA, ${ }^{52}$ Center for Research and Exploration in Space Science and Technology, NASA/GSFC, Greenbelt, MD, USA, ${ }^{53}$ Instituto de Física Corpuscular, CSIC, Universitat de València, Paterna, Valencia, Spain 